\title{
An Evolutionary Approach for LAUC Scheduler in Optical Burst Switching Networks
}

\author{
Shalini V. Wankhade \\ Sinhgad Academy Of Engg. \\ Kondhawa, Pune
}

\author{
Swarupa B. kambale \\ Sinhgad Institute Of Tech. and Science Narhe, \\ Pune
}

\begin{abstract}
Optical Burst Switching (OBS) has been proposed as a promising switching technology for the next generation of optical transport network. OBS is a technology positioned between Optical Circuit Switching (OCS) and Optical Packet Switching (OPS) that does not require optical buffering, and it is more efficient than circuit switching when the sustained traffic volume does not consume a full wavelength. OBS uses various scheduling algorithms like LAUC, LAUCVF, LAUC Min, LAUC max for assignment of channels for data transmission. By applying evolutionary techniques we will improve the performance of LAUC scheduler.
\end{abstract}

\section{General Terms}

Optical Burst Switching Networks, Genetic, Evolutionary Methods.

\section{Keywords}

OBS, OPS, OCS, LAUC, LAUCVF.

\section{INTRODUCTION}

The demand for network bandwidth has been increasing remarkably due to the increasing demands of Internet. Dramatically increased amount of the World Wide Web users have brought more information servers on line. Again the types of network services have been largely increased, and the proportion of multimedia technologies integrated by video and audio becomes larger. The Internet traffic increase rapidly, and the demand for network bandwidth becomes more urgent than ever. With the explosive growth of the Internet and the rapid evolution of Wavelength Division Multiplexing (WDM) technique, optical fiber seems to be the perfect carrier for future high-speed networks.

In a WDM networks, each fiber carries multiple communication channels, with each channel operating on a different wavelength. Such an optical transmission system has a potential capacity to provide over $50 \mathrm{Tbps}$ bandwidth on a single fiber. Apart from this, optical fiber is inexpensive and provide extremely low bit-error rate (typically $10 ; 12$ ). The optical fiber is less bulky than other cables. Optical signals travel clearly for longer distances and are immune to electrical interferences. Further more, fiber cables are much more difficult to tap than copper wires, so in addition there is a security advantage. All these factors make optical data networks the networks of the future. To realize an IP-overWDM architecture, several approaches, such as Optical Circuit Switching (OCS), Optical Packet Switching (OPS) and Optical Burst Switching (OBS) have been proposed. In all these approaches, OBS can achieve a good balance between the coarse-gained OCS and fine-gained OPS. Internet traffic has grown by leaps and bounds in the recent past. Fiber optic networks are extensively used for handling this huge volume of data. Traditional methods for transmitting data over a optic fiber network involve optic-electronic-optic conversion $(\mathrm{O} / \mathrm{E} / \mathrm{O})$. In this method when data in optical mode reaches a node in the network the data is converted into electrical signals for the purpose of processing. After the processing is over the data may or may not be converted to optical form for further routing. This process is time consuming and leads to wastage of energy. An alternative to this technique is "All Optical Switching". In this method there is no conversion of optical signals to electrical signals. All the processing is done using optical signals only. Current networks typically consist of four layers: IP layer for carrying applications and services, Asynchronous Transfer Mode (ATM) layer for traffic engineering, SONET/SDH layer for transport, and WDM for capacity.

When the data stream arrives at an optical switching point, the optical signal of data is converted into electronic form, and the processing and forwarding are done in the electronic domain. This is known as an Optical-Electronic $(\mathrm{O} / \mathrm{E})$ conversion. When the electronic signal of data is passed to the output port, it is again converted and modulated into the fiber as an Electronic-Optical signal (E/O). Such a switching point is said to perform $\mathrm{O} / \mathrm{E} / \mathrm{O}$ conversion. In such a network, all communication is limited by the electronic processing capabilities of the system. Although hardware-based highspeed electronic IP routers with capacity up to a few hundred gigabits per second are available now, there is still a serious mismatch between the transmission capacity of WDM fibers and the switching capacity of electronic IP routers. Next generation of optical network is optical-packet-switchednetwork (OPSN). OPSN is the most sophisticated and seem to be an ideal architecture for future optical networks. As clear from name, the traffic in OPSN is carried in optical packets along with in-band control information, and problem with OPSN is the lack of optical logic and practical optical buffer $[3,5,7]$. An alternative to the OCS and OPS is OBS. In OBS, packets are concatenated into transport units referred to as burst. In OBS, user data travels entirely in the optical domain. The elimination of $\mathrm{O} / \mathrm{E} / \mathrm{O}$ conversion in OBS allows unprecedented transmission rate. With respect to current technology presently OBS is the most suitable AON control framework. It combines the best feature of both circuit switching and packet switching.

\section{OPTICAL SWITCHING TECHNOLOGIES}

\subsection{Optical Circuit Switching (OCS)}

In OCS, a dedicated path is established between the source and the destination before data transmission begins. During data transmission, there is no need for the intermediate nodes to perform complex processing of packet header and buffering of the payload. The reserved resources stay idle for the entire path setup time and account for poor resource utilization. One 
of the main feature of OCS is its two-way reservation process in set-up of lightpath, where a source sends a request for setting up a lightpath and then receives an acknowledgement back from the corresponding destination [4].

\subsection{Optical Packet Switching (OPS)}

OPS are suitable for supporting busty traffic since it allows statistical sharing of the wavelengths among packets belonging to different source and destination pairs. When an optical packet arrives, the optical core node first departs the header from the payload and converts it into electronic domain and processes it electronically Packet switching has large buffer requirement. For the optical domain, packet switching is not yet feasible because of optical hardware limitations. Optical RAMs do not exist yet to meet the high buffer requirements of packet.[6]

\subsection{Optical Burst Switching (OBS)}

OBS is an optical networking technique that allows dynamic sub-wavelength switching of data. Data is transported in various size units called bursts. OBS is viewed as a compromise between the yet unfeasible full optical packet switching (OPS) and the mostly static optical circuit switching (OCS). It differs from these paradigms because OBS control information is sent separately in a reserved optical channel and in advance of the data payload. In the OBS paradigm, only few control channels go through $\mathrm{O} / \mathrm{E} / \mathrm{O}$ conversion and there is a strong separation between control and data planes. This allows great network manageability and flexibility. Since, OBS takes advantages of both the huge capacity in fibers for switching/transmission and the sophisticated processing capability of electronic devices, which makes it a viable technology for the next generation optical network.

In OBS, packets are aggregated into data bursts at the edge of the network to form the data payload. Various assembling schemes based on time and size exist. OBS features the separation between the control plane and the data plane. A control signal (also termed burst header or control packet) is associated to each data burst. The control signal is transmitted in optical form in a separated wavelength termed the control channel, but signaled out of band and processed electronically at each OBS router, whereas the data burst is transmitted in all optical form from one end to the other end of the network. The data burst can cut through intermediate nodes, and data buffers such as fiber delay lines may be used. In OBS data is transmitted with full transparency to the intermediate nodes in the network. After the burst has passed a router, the router can accept new reservation requests.

\subsection{Processes in OBS}

Burst Assembly/ Disassembly:- At the edge and core node it takes place.

Wavelength Reservation Protocols:- A control packet is sent ahead to reserve wavelength along its route for its data burst using Tell And Wait and Tail And Go protocols

Scheduling Algorithms An incoming burst may be scheduled onto multiple wavelengths at the desired output port burst scheduler takes care of that.

Contention Resolution In a bufferless OBS network, contention among the bursts can be resolved in three ways: deflection, dropping and preemption.

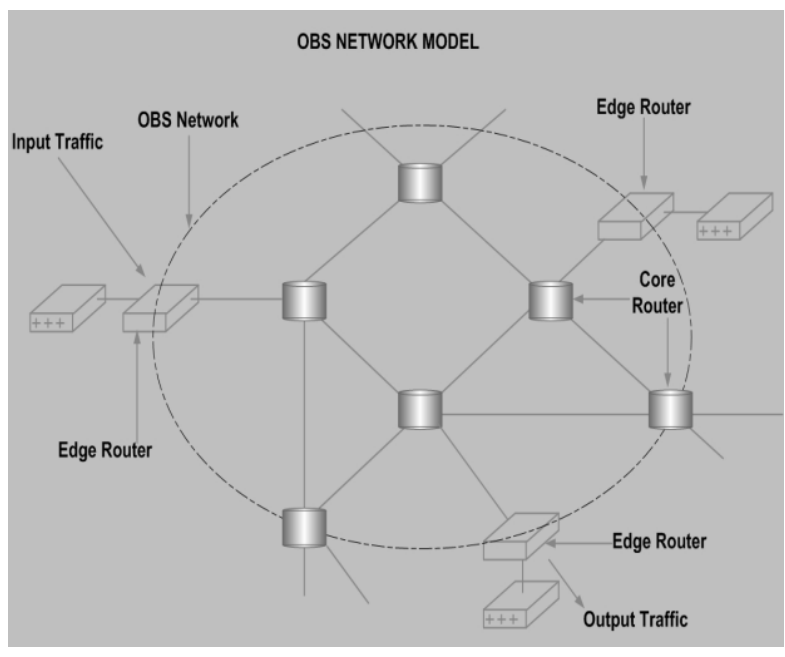

\section{BURST SCHEDULING ALGORITHMS}

As the arrival of bursts at OBS node is dynamic. When a CP arrives at a core node, the core node converts it into electronic signal and obtains the burst arrival time and duration of its corresponding data burst from the CP. Scheduling technique must schedule arrival burst on the available wavelength for the entire duration of burst transmission efficiently and quickly. The optical switching fabric is reconfigured based on the scheduling results. Scheduling algorithm should able to process the $\mathrm{CP}$ fast enough before the burst arrives to the node and also scheduling algorithm able to find proper void for an incoming burst to increase channel bandwidth utilization ratio. The algorithms are like Latest Available Unused Channel (LAUC) AlgorithmLatest Available Unused Channel with Void Filling (LAUC-VF) Algorithm Minimum Starting Void Filling (Min-SV) Algorithm Minimum Ending Void Filling (Min-EV) Algorithm Best Fit (BF) Algorithm.

\subsection{Latest Available Unused Channel (LAUC) Algorithm}

In LAUC, bursts scheduling is done by selecting the latest available unscheduled data channel for each arriving data burst. In this algorithm, scheduler keeps track of horizon for each data channel. Horizon is the time after which no reservation has been made on that data channel. The scheduler assigns each arriving burst to the channel with minimum void formed by that burst on data channel [6].

The pseudo-code of the algorithm is given below. The new burst is assumed to arrive at time $t$. The latest available time of wavelength channel $\mathrm{Ci}$ is denoted by $t i$.

Begin

1. Channel Searching: For every wavelength $\mathrm{Ci}$ compute $m i$ as $t$-ti if $t i \cdot t$; otherwise set $m i$ to infinity.

2. Channel Selection: Choose wavelength $C j$ such that $m j$ is finite and is minimum among all. Assign wavelength $C j$ to the new burst.

3. Burst Dropping: If no such $C j$ exists, then drop the burst. End. 


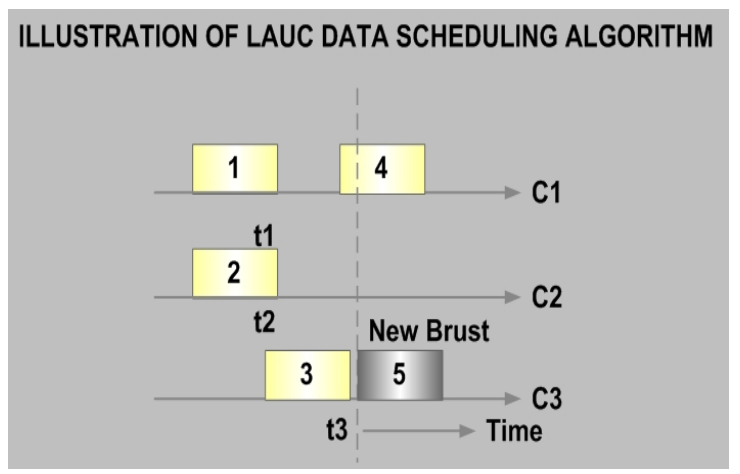

Fig. 2: Illustration of LAUC Data Scheduling Algorithm

For example, in Fig.2, wavelength $C 2$ and $C 3$ is unscheduled at the arrival time $t$ of the new burst. Wavelength $C 3$ will be selected for the new burst because the generated void $(t-t 3)$ on wavelength $C 3$ will be smaller than the void $(t-t 2)$ that would have been created if wavelength $C 2$ was selected. LAUC algorithm is simple and has a good performance in terms of its execution time. However, it results in low bandwidth utilization and a high burst loss rate.

\subsection{Evolutionary Algorithms}

Evolutionary algorithms are population-based meta-heuristic optimization algorithms that use biology-inspired mechanisms like mutation, crossover, natural selection, and survival of the fittest in order to refine a set of solution candidates iteratively. The advantage of evolutionary algorithms compared to other optimization methods is their "black box" character that makes only few assumptions about the underlying objective functions. Furthermore, the definition of objective functions usually requires lesser insight to the structure of the problem space than the manual construction of an admissible heuristic. EAs therefore perform consistently well in many different types of problems. LAUC (Latest unavailable unscheduled channel) is an algorithm which is used in OBS for assigning channels for data transmission. The standard implementation of LAUC uses the concept of "Horizon". "Horizon" indicates the latest time at which a particular wavelength will be free. LAUC assigns incoming bursts to channels with maximum "Horizon" values. We will use Evolutionary algorithms to optimize the assignment of channels to "burst". Given a set of data of different sizes and priority and a fixed set of channels we will devise an algorithm which will output an optimal matrix for scheduling of data transmission

\subsubsection{Algorithm details for LAUC scheduler optimization}

We will optimize the LAUC scheduler which will maximize the packet throughput rate with respect to a given packet size and data link capacity. We will implement the following algorithm for this purpose:

Algorithm $: \mathrm{X} \leftarrow \operatorname{simpleEA}(\mathrm{cmpF}, \mathrm{ps})$
Input: $\mathrm{cmpF}$ : the comparator function which allows us to compare the utility of two solution candidates

Input: ps: the population size

Data: $t$ : the generation counter

Data: Pop: the population

Data: Mate: the mating pool

Data: v: the fitness function resulting from the fitness assigning process

Output: X: the set of the best elements found

\section{1 begin}

$2 \mathrm{t} \leftarrow-0$

3 Pop $\leftarrow$ createPop(ps)

4 while $\neg$ terminationCriterion() do

$5 \mathrm{v} \leftarrow \operatorname{assignFitness}(\mathrm{Pop}, \mathrm{cmpF})$

6 Mate $\leftarrow \operatorname{select}($ Pop, v, ps)

$7 \mathrm{t} \leftarrow \mathrm{t}+1$

8 Pop $\leftarrow$ reproducePop(Mate)

9 return extractPhenotypes(extractOptimalSet(Pop))

10 end

1. Initially, a population Pop of $\mathrm{p}$ number of packets with random sizes and $n$ number of data link connections with different capacities will be created.

2. The values of the objective functions $\mathrm{f} \leftarrow \mathrm{F}$ are computed for each solution candidate $\mathrm{p}(\mathrm{i}) \cdot \mathrm{x}+\mathrm{n}(\mathrm{i}) \mathrm{x}$. The objective function in this case will be packet throughput rate.

3. With the objective functions, the utility of the different features of the solution candidates have been determined and a fitness value $v(p(i) \cdot x+n(i) x)$ can now be assigned to each of them. This fitness assignment process can, for instance, incorporate a prevalence comparator function $\mathrm{cmpF}$ which uses the objective values to create an order amongst the individuals.

4 A subsequent selection process filters out the solution candidates with bad fitness and allows those with good fitness to enter the mating pool with a higher probability. Since fitness is subject to minimization in the context of this book, the lower the $\mathrm{v}(\mathrm{p}(\mathrm{i}) \cdot \mathrm{x}+\mathrm{n}(\mathrm{i}) \mathrm{x})$-values are, the higher is the (relative) utility of the individual to whom they belong.

5. In the reproduction phase, offspring is created by varying or combining the genotypes p.g of the selected individuals 
$p(i) \cdot x+n(i) x$ Mate by applying the search operations searchOp Op (which are called reproduction operations in the context of EAs). These offspring are then subsequently integrated into the population.

6. If the terminationCriterion () is met, the evolution stops here. Otherwise, the algorithm continues at step 2

This is the proposed system to improve the performance of LAUC scheduler by using genetic technique like evolutionary approach.

So this is the technique by which we can improve the performance of LAUC Scheduler. When the incoming burst comes, for assigning the channel to the respective burst we can use heuristic approach. The genetic comes into picture in that we can apply the evolutionary method for assignment of channel. We can also improve the performance by applying simulated annealing also.

\section{ACKNOWLEDGMENTS}

For the contribution of this paper our thanks is to the experts team members for their support.

\section{REFERENCES}

[1] Time Sliced Optical Burst Switching, Jeyashankher Ramamirtham, Jonathan Turner, Computer Science and Engineering Department, Washington University in St. Louis, IEEE Infocom 2003

[2] Performance Analyses of Optical Burst-Switching Networks, Zvi Rosberg, Hai Le Vu, Moshe Zukerman, and Jolyon White, IEEE JOURNAL ON SELECTED AREAS IN COMMUNICATIONS, VOL. 21, NO. 7, SEPTEMBER 2003

[3] Teletraffic Performance Models for All-Optical Networks and their Analysis, Phd Thesis, Andrew L.
Zalesky, Department of Electrical and Electronic Engineering University of Melbourne, Australia,2006 B. Mukharjee, Optical WDM Networks, Springer Publication, 2006.

[4] Basem Shihada and Pin-Han Ho, Transport Control Protocol in Optical Burst Switched Networks: Issues,Solutions, and Challenges", IEEE Communication Surveys and Tutorials, vol. 10, 2nd Quarter 2008.

[5] Tzvetelina Battestilli and Harry Perros, \An Introduction to Optical Burst Switch-ing", IEEE Optical Communication, Aug. 2003.

[6] C.Qiao and M. Yoo, IOptical Burst Switching: A New Paradigm For An Optical Internet", Journal of High Speed Network, vol. 8, pp. 69-84, 1999.

[7] Tzvetelina Battestilli, IOptical Burst Switching: A Survey", Technical Report, NC State University, Computer Science Department, July 2002.

[8] B.Praveen, J.Praveen and C. Siva Ram Murty, A Survey of di®erentiated QoSschemes in optical burst switched networks", SCIENCE DIRECT Optical Switching and Networking, vol. 3, pp. 134-142, July 2006.

[9] Jinhui Xu., Chunming Qiao, Jikai Li and Guang Xu, Efficient Channel Scheduling Algorithms in Optical Switched Networks using Geometric Technique", IEEE Journal on selected areas in Communication, vol. 22, No. 9, November 2004.

[10] S. K.Tan, G.Mohan and K.K.Chua, Burst Rescheduling with Wavelength and Last-hop FDL Reassignment in WDM Optical Burst Switching Networks", IEEE Computer Networks, 2003.

[11] S .K.Tan, G.Mohan and K. K.Chua, Algorithms for Burst Rescheduling in WDM Optical Burst Switching Networks", IEEE Computer Networks, pp. 41-55, 2002. 